\title{
A PEANO CONTINUUM HOMEOMORPHIC TO ITS OWN SQUARE BUT NOT TO ITS COUNTABLE INFINITE PRODUCT
}

\author{
JAN VAN MILL
}

Abstract. We give an example of a Peano continuum $X$ with $X^{2} \approx X$ but $X^{\infty} \approx X$.

In [2], Fajtlowicz, Holsztyński, Mycielski and Wȩglorz ask whether there is a (metrizable) continuum $X$ with $X^{2} \approx X$ (as a consequence, $X^{n} \approx X$ for all $n \in \mathrm{N}$ ) but $X^{\infty} \approx X$. The aim of this note is to construct a Peano continuum with these properties. Our basic tool is a simple but interesting result due to van Douwen [1, 17.1]: if $X$ is a locally compact space homeomorphic to its own square, then $X$ has a compactification homeomorphic to its own square.

Let $E$ be the topological product of countably many copies of the 1-sphere $S^{1}$ and let $B$ be the topological sum of $\mathbf{N} \times E$ and a one-point space. Clearly $B^{2} \approx B$. As usual, $I$ denotes $[0,1]$ and $Q$ denotes the Hilbert cube. Put $Y=B \times[0,1) \times Q$.

LEMMA $1 . Y^{2} \approx Y$.

Proof. $\quad Y^{2}=(B \times[0,1) \times Q) \times(B \times[0,1) \times Q) \approx(B \times B) \times([0,1) \times$ $[0,1)) \times(Q \times Q) \approx B \times([0,1) \times I) \times Q \approx B \times[0,1) \times Q=Y$.

Since $Y$ is clearly locally compact, by van Douwen's result previously cited, $Y$ has a compactification homeomorphic to its own square. It turns out that the compactification of $Y$ we get from the proof of van Douwen's result is a Peano continuum $X$ not homeomorphic to its countable infinite product. For the reader's convenience we repeat van Douwen's argument and give the proof that the resulting compactification is Peano.

LEMMA 2. $Y$ has a Peano compactification $X$ with $X^{2} \approx X$.

Proof. The compactification $X$ of $Y$ is the inverse limit of a suitable inverse sequence $\left(a_{n} Y, f_{n}\right)_{n}$ of compactifications $a_{n} Y$ of $Y$, where for each $n \in \mathrm{N}, f_{n}$ is a mapping from $a_{n+1} Y$ onto $a_{n} Y$ with $f_{n} \mid Y=\mathrm{id}_{Y}$. As is well known, and easy to prove, this implies that $f_{n}\left(a_{n+1} Y-Y\right)=a_{n} Y-Y$. In order to show that $X^{2} \approx X$ we will construct the inverse sequence in such a way that for each $n \in \mathbf{N}$ there is a homeomorphism $h_{n}: a_{n+1} Y \rightarrow a_{n} Y \times a_{n} Y$ so that the diagram

Received by the editors March 12, 1980.

AMS (MOS) subject classifications (1970). Primary 54F25, 54B25, 54B10.

Key words and phrases. Peano continuum, countable infinite product, cell-like map. 


$$
\begin{array}{ccccc}
a_{1} Y \stackrel{f_{1}}{\leftarrow} & \begin{array}{c}
a_{2} Y \\
h_{1} \downarrow
\end{array} & \stackrel{f_{2}}{\leftarrow} & a_{3} Y & \leftarrow \\
& & h_{2} \downarrow & \\
& a_{1} Y \times a_{1} Y & \stackrel{f_{1} \times f_{1}}{\leftarrow} & a_{2} Y \times a_{2} Y & \leftarrow
\end{array}
$$

commutes. As remarked by van Douwen [1, 17.1], the construction is straightforward. Let $a_{1} Y$ be the one-point compactification of $Y$ and let $h$ be any homeomorphism from $Y$ onto $Y^{2}$ (Lemma 1). There is a compactification $a_{2} Y$ of $Y$ so that $h$ extends to a homeomorphism $h_{1}: a_{2} Y \rightarrow a_{1} Y \times a_{1} Y$. Since $a_{1} Y$ is the smallest compactification of $Y$, the identity mapping on $Y$ extends to a mapping $f_{1}$ : $a_{2} Y \rightarrow a_{1} Y$. Similarly, there is a compactification $a_{3} Y$ of $Y$ such that $h$ extends to a homeomorphism $h_{2}: a_{3} Y \rightarrow a_{2} Y \times a_{2} Y$. Define $f_{2}: a_{3} Y \rightarrow a_{2} Y$ by $f_{2}=h_{1}^{-1}$ 。 $\left(f_{1} \times f_{1}\right) \circ h_{2}$. It is easily seen that $f_{2} \mid Y=\mathrm{id}_{Y}$. In the same way we define $a_{4} Y, h_{3}$ and $f_{3}$, etc.

Clearly $X=\lim _{\leftarrow}\left(a_{n} Y, f_{n}\right)_{n}$ is a compactification of $Y$ homeomorphic to its own square. It remains to be shown that $X$ is Peano. First observe that $a_{1} Y$ is Peano. This implies that $a_{2} Y$ is Peano, being homeomorphic to $a_{1} Y \times a_{1} Y$. Continuing in this way, we see that all the $a_{n} Y$ 's are Peano continua. So $X$ is the inverse limit of an inverse sequence of continua, hence $X$ is itself a continuum. It remains to be shown that $X$ is locally connected. From the local connectivity of the $a_{n} Y$ 's it is easy to derive that $X$ is locally connected provided that the bonding maps $f_{n}$ are all monotone. But this is clear from the construction.

We will now show that $X^{\infty} \approx X$.

LEMMA 3. $X^{\infty} \approx X$.

Proof. Suppose, to the contrary, that $h$ is a homeomorphism from $X$ onto $X^{\infty}$. Since $Y$, being locally compact, is open in $X, h(Y)$ is open in $X^{\infty}$. In addition, since $B$ has an isolated point, $Y$ contains an open copy of $[0,1) \times Q$. Therefore, $X^{\infty}$ contains an open copy of $[0,1) \times Q$, say $F$. Since $F$ is open and nonempty, we can find an integer $n$ and open nonempty sets $U_{i} \subset X(i<n)$ so that

$$
U_{1} \times U_{2} \times \cdots \times U_{n} \times X \times X \times \cdots \subset F .
$$

Hence $X$ is (homeomorphic to) a retract of some open subspace of $F$, or, equivalently, $X$ is homeomorphic to a retract of some open subset of $[0,1) \times Q$. Since $[0,1) \times Q$ is locally contractible, this implies that $X$ is locally contractible. Then $Y$, and therefore the countable infinite product of circles, is locally contractible, a contradiction.

It might be worthwhile to notice that our example has trivial shape, since it is the inverse limit of an inverse sequence consisting of contractible continua. This is of interest, since if $X$ is a nondegenerate AR which is compact and homeomorphic to its own square, then $X$ is homeomorphic to the Hilbert cube, [4], in which case $X$ is homeomorphic to its countable infinite product.

Let us also remark that Taylor's [3] Example can be used instead of the 1-sphere $S^{1}$. By a double inverse limit construction one can then produce a cell-like map $f$ : $Q \rightarrow Y$ so that $Y$ has trivial shape, $Y^{2} \approx Y$ but $Y^{\infty} \approx Y$. This might be of interest, 
since there is a topological, and not algebraic, argument why $Y$ is not an AR. If $Y$ were an AR then, by Torunczyk's [4] result, $Y \approx Q$, so $Y^{\infty} \approx Y$, which is not the case. We will sketch the construction.

Let $f: Q \rightarrow X$ be a cell-like map which is not a hereditary shape equivalence. We may assume that $X^{\infty} \approx X$. Let $B$ be the topological sum of $\mathbf{N} \times X$ and a one-point space. Similarly, let $B_{0}$ be the topological sum of $\mathbf{N} \times Q$ and a one-point space. Define $\hat{f}: B_{0} \stackrel{\text { onto }}{\rightarrow} B$ so that $\hat{f}(n, q)=(n, f(q))$ for all $(n, q) \in \mathbf{N} \times Q$. Put $E=B \times$ $[0,1) \times Q$ and $E_{0}=B_{0} \times[0,1) \times Q$. There clearly exist, by Lemma 1 , a homeomorphism $h: E \rightarrow E^{2}$ and a homeomorphism $h_{0}: E_{0} \rightarrow E_{0}^{2}$ so that the diagram

$$
\begin{array}{rll}
E & \stackrel{h}{\rightarrow} & E^{2} \\
\hat{f} \times \mathrm{id} \uparrow & & \uparrow(\hat{f} \times \mathrm{id}) \times(\hat{f} \times \mathrm{id}) \\
E_{0} & \stackrel{h_{0}}{\rightarrow} & E_{0}^{2}
\end{array}
$$

commutes. We can then, using these homeomorphisms, run both $E$ and $E_{0}$ through the inverse limit construction of Lemma 2. It is routine to verify that all bonding maps are cell-like and that the inverse system we obtain from $E_{0}$ consists of AR's. The second inverse limit maps onto the first inverse limit by a cell-like map (since the bonding maps in the second inverse system are hereditary shape equivalences). The second inverse limit is an AR homeomorphic to its own square, so it is homeomorphic to the Hilbert cube. The first inverse limit $Y$ is homeomorphic to its own square and it can be shown that $Y$ is not homeomorphic to its countable infinite product by a method similar to the one used in the proof of Lemma 2 by noting that $X$ is not an ANR.

\section{REFERENCES}

1. E. K. van Douwen, Prime mappings, number of factors, and binary operations, Dissertationes Math. (to appear).

2. S. Fajtlowicz, W. Holsztyński, J. Mycielski and B. Weglorz, On powers of bases in some compact algebras, Colloq. Math. 19 (1968), 43-46.

3. J. L. Taylor, A counterexample in shape theory, Bull. Amer. Math. Soc. 81 (1975), 629-632.

4. H. Torunczyk, Characterization of infinite-dimensional manifolds (Proc. Geom. Topol. Conf., Warsaw, 1978) (to appear).

Department of Mathematics, Louisiana State University, Baton Rouge, Louisiana 70803

Current address: Subfaculteit Wiskunde, Vrije Universiteit, De Boelelaan 1081, Amsterdam, The Netherlands 